\title{
The Missing Satellites of the Magellanic Clouds? Gaia Proper Motions of the Recently Discovered Ultra-faint Galaxies
}

\author{
Nitya Kallivayalil ${ }^{1}$ (D), Laura V. Sales ${ }^{2}$, Paul Zivick ${ }^{1}$ (10), Tobias K. Fritz ${ }^{3,4}$, Andrés Del Pino ${ }^{5}$, Sangmo Tony Sohn ${ }^{5}$ (ID, \\ Gurtina Besla $^{6}$, Roeland P. van der Marel $^{5,7}$ (D), Julio F. Navarro ${ }^{8,9}$, and Elena Sacchi ${ }^{5}$ (D) \\ ${ }^{1}$ Department of Astronomy, University of Virginia, 530 McCormick Road, Charlottesville, VA 22904, USA; njk3r@ virginia.edu \\ ${ }^{2}$ Department of Physics and Astronomy, University of California Riverside, 900 University Avenue, CA 92507, USA \\ ${ }^{3}$ Instituto de Astrofisica de Canarias, Calle Via Lactea s/n, E-38205 La Laguna, Tenerife, Spain \\ ${ }^{4}$ Universidad de La Laguna, Dpto. Astrofisica, E-38206 La Laguna, Tenerife, Spain \\ ${ }^{5}$ Space Telescope Science Institute, 3700 San Martin Drive, Baltimore, MD 21218, USA \\ ${ }^{6}$ Steward Observatory, University of Arizona, 933 North Cherry Avenue, Tucson, AZ 85721, USA \\ ${ }^{7}$ Center for Astrophysical Sciences, Department of Physics and Astronomy, Johns Hopkins University, Baltimore, MD 21218, USA \\ ${ }^{8}$ Department of Physics and Astronomy, University of Victoria, Victoria, BC V8P 5C2, Canada \\ Received 2018 May 7; revised 2018 August 6; accepted 2018 September 4; published 2018 October 25
}

\begin{abstract}
According to LCDM theory, hierarchical evolution occurs on all mass scales, implying that satellites of the Milky Way should also have companions. The recent discovery of ultra-faint dwarf galaxy candidates in close proximity to the Magellanic Clouds provides an opportunity to test this theory. We present proper motion (PM) measurements for 13 of the 32 new dwarf galaxy candidates using Gaia data release 2. All 13 also have radial velocity measurements. We compare the measured 3D velocities of these dwarfs to those expected at the corresponding distance and location for the debris of a Large Magellanic Cloud (LMC) analog in a cosmological numerical simulation. We conclude that four of these galaxies (Hor1, Car2, Car3, and Hyi1) have come in with the Magellanic Clouds, constituting the first confirmation of the type of satellite infall predicted by LCDM. Ret2, Tuc2, and Gru1 have velocity components that are not consistent within $3 \sigma$ of our predictions and are therefore less favorable. Hya2 and Dra2 could be associated with the LMC and merit further attention. We rule out Tuc3, Cra2, Tri2, and Aqu2 as potential members. Of the dwarfs without measured PMs, five of them are deemed unlikely on the basis of their positions and distances alone being too far from the orbital plane expected for LMC debris (Eri2, Ind2, Cet2, Cet3, and Vir1). For the remaining sample, we use the simulation to predict PMs and radial velocities, finding that Phx2 has an overdensity of stars in DR2 consistent with this PM prediction.
\end{abstract}

Key words: dark matter - galaxies: interactions - galaxies: kinematics and dynamics - Local Group - Magellanic Clouds - proper motions

\section{Introduction}

In the prevailing $\Lambda$ Cold Dark Matter cosmology (LCDM), dark matter (DM) halos build up their mass by the accretion of smaller objects. The satellites that orbit our Milky Way (MW) are remnants of this process, giving us insights into the accretion events that built our Galaxy's DM halo. Selfsimilarity dictates that this hierarchical evolution should occur on all mass scales, implying that satellites of the MW also once had companions. However, testing this picture is challenging - faint companions of dwarf galaxies are difficult to detect at large distances and the tidal field of the MW acts to disrupt such groups over multiple orbits. The recent discoveries of 32 candidate low mass, dwarf galaxies in close proximity on the sky to the Magellanic Clouds (Bechtol et al. 2015; Drlica-Wagner et al. 2015, 2016; Kim \& Jerjen 2015; Kim et al. 2015; Koposov et al. 2015a, 2018; Laevens et al. 2015a; Martin et al. 2015; Torrealba et al. 2016a, 2016b, 2018; Homma et al. 2018) present a new opportunity to test this theory.

Our understanding of the orbital history of the Magellanic Clouds has also gone through a recent revision. The Clouds are moving faster with respect to the MW than previously believed (Kallivayalil et al. 2006a, 2006b, 2013), implying that, instead

\footnotetext{
${ }^{9}$ Senior CIfAR Fellow.
}

of being long-term companions, they are likely on their first infall toward the MW (Besla et al. 2007). Such late infall is not unexpected within the LCDM paradigm (Boylan-Kolchin et al. 2011). This scenario further implies that MW tides have not had sufficient time to disrupt the infalling system, and so companions of the Clouds should share common orbital properties (Sales et al. 2011, 2017, hereafter S17).

A massive satellite such as the Large Magellanic Cloud (LMC) is expected to host its own satellites at infall (e.g., Lynden-Bell 1976; D’Onghia \& Lake 2008; Li \& Helmi 2008). Using semi-analytic models of galaxy formation, Sales et al. (2013) determined the number of satellites expected around dwarf galaxies like the LMC in LCDM theory (see also Guo et al. 2010, 2011). The LMC has a stellar mass of $M_{\star} \sim 3 \times 10^{9} M_{\odot}$ (van der Marel et al. 2002), implying a DM mass of $\log \left(M_{\text {dark }}\right)=10.75-11.25$ prior to capture by the MW (Kallivayalil et al. 2013; Moster et al. 2013). From Sales et al. (2013), such LMC analogs should host one Small Magellanic Cloud analog (mass ratio $\sim 0.1$ ) in addition to $\sim 5-40$ satellites that are $\times 0.001$ its mass, i.e., ultra-faint dwarf galaxies: $M_{\star} \sim(0.1-1) \times 10^{4} M_{\odot}, \log \left(M_{\text {dark }}\right) \sim 8$. This begs the question: where are these galaxies today?

Wetzel et al. (2015) used the ELVIS suite of cosmological simulations of MW analogs (Garrison-Kimmel et al. 2014) to trace the orbital histories of surviving satellites at $z=0$. They determined that half of the current low-mass $\left(M_{\star}<10^{6} M_{\odot}\right)$ 
satellites of the MW were preprocessed in a group prior to capture by the MW. The identification of the surviving satellite population of the LMC provides a crucial testing ground for such theories, and for the halo occupation function at low-mass scales.

The discovery of dwarf galaxies in the vicinity of the Clouds could help answer these questions. The newly discovered dwarfs range in visual magnitudes from $M_{V}=-2$ to -8 , and have halflight radii $r_{h} \sim 20-1100 \mathrm{pc}$. While some of these candidates have yet to be confirmed as DM-dominated galaxies using velocity dispersion measurements, they generally have magnitudes and sizes consistent with those of known ultra-faint dwarf galaxies orbiting the MW and M31 (McConnachie 2012).

A few past works have attempted to assess membership of these candidates to the Clouds. Deason et al. (2015) used a statistical argument based on abundance-matching models applied to massive subhalos in the ELVIS simulations to suggest that two to four of the nine then known candidates might have come into the MW with the LMC. Yozin \& Bekki (2015), on the other hand, conclude, on the basis of orbit models, that the majority of those dwarfs could have been at least loosely associated with the Clouds. Jethwa et al. (2016) constructed a dynamical model for the Magellanic Clouds satellite population based on numerical simulations. They compared this to the observed 3D spatial distribution of the candidate dwarfs, excluding likely globular clusters. They inferred that at $1 \sigma$ confidence, $50 \%$ of the candidates have $>70 \%$ probability of a Magellanic Cloud's association. Dooley et al. (2017) used the abundance-matching method to determine what fraction of the new ultra-faint dwarfs could be associated to the LMC depending on the assumed relation between stellar mass and halo mass.

In a different approach, S17 (using an extension of the setup in Sales et al. 2011) identified an LMC analog in a fully cosmological simulation of a MW-sized halo in LCDM. They tracked the positions and velocities of subhalo particles to constrain the likely location in phase space of systems with prior association with the LMC, finding that of the six systems with kinematic data at the time, only Hydra II and Hor 1 had distances and radial velocities consistent with a Magellanic origin. Of the remaining dwarfs, six (Hor 2, Eri 3, Ret 3, Tuc 4, Tuc 5, and Phx2) had positions and distances consistent with a Magellanic origin, but kinematic data were needed to substantiate that possibility. Conclusive evidence for association would require proper motions (PMs) to constrain the orbital angular momentum direction, which, for true Magellanic satellites, must coincide with that of the Clouds.

Here, we measure PMs from data release 2 (DR2) of the Gaia survey (Gaia Collaboration et al. 2018a) for 13 of the 32 newly discovered dwarfs. We combine these PMs with the measured radial velocities from other studies, and compare to the predictions from the Sales et al. (2017) set up to confirm or rule out the dynamical association of these dwarfs with the Magellanic system.

In Section 2, we describe our methodology to select member stars for each of the dwarfs and to measure their PMs. In Section 3, we compare the resultant Galactocentric velocities to that of LMC debris at the positions and distances of these dwarf galaxies in the simulation. We discuss and conclude in Section 4.

\section{Membership Selection and Proper Motion Determination}

We retrieved data within three times the half-light radii of each dwarf galaxy (see Table 1) from the Gaia archive using pygacs. ${ }^{10}$ Following Gaia Collaboration et al. (2018b), we first clean the source lists of stars with visibilityperiods-used $<5$ and relatively well-measured parallaxes, indicative of MW foreground stars, using $\omega-2 \sigma_{\omega}>0$.

We next consider a set of nested criteria for our membership selection based on the publicly available spectroscopic member catalogs, the PMs, and the position in the color-magnitude diagram (CMD). We start with the sample of spectroscopic target lists compiled and presented in Fritz et al. (2018). These lists contain stars that were deemed spectroscopic members as well as nonmembers by the original authors. We then pull stars from our DR2 source lists that match the weighted-average PMs of the spectroscopic members to within $2 \sigma$, where $\sigma$ is the variance. PARSEC isochrones (Bressan et al. 2012), with ages and metallicities compiled from the literature, are then used to define a region in the CMD where member stars are expected to populate it. Only stars whose position in the CMD is compatible with the used isochrones are considered as possible members. We use $2 \sigma$ as the maximum allowed distance from the star to the isochrone. We then perform these same steps on a "background" field, chosen to have the same galactic longitude as the dwarf under consideration, but opposite galactic latitude. Stars in the background field that pass the same PM and CMD cuts as the target field are used to construct a "Hess" diagram as follows: since we are dealing with quite sparse CMDs in general, we choose a fairly coarse grid of $5 \times 5$ bins. We then use the total number of background stars in the CMD and this number of bins to set an average density. This average density is assigned to any bin that contains zero stars. Then for each target star we interpolate over the four closest bins to assign an expected density at that point. We then use this density to weight the PM error of each star, i.e., multiply the PM errors by the ratio of the average to the expected density.

Unsurprisingly, we pick up many potential member stars in fields closer to the disk. We therefore impose a strict additional cut that any new members must be within a radius of $1 r_{\mathrm{h}}$ of the dwarf center. This rejects many stars and cuts out the possibility of detecting any tidal signatures, but here we are aiming only for accurate center-of-mass PMs, and hence choose to focus on the cores of the dwarfs. At the end of this procedure, we ensure that our newly minted members have not already been targeted and rejected as nonmembers in the original spectroscopic samples.

In summary, the method involves the following steps: (1) obtain clean source lists within $3 r_{\mathrm{h}}$ of each dwarf; (2) clean for parallax following Gaia Collaboration et al. (2018b); (3) obtain an initial set of candidate member stars using the weighted-average PM of the spectroscopic members plus or minus $2 \sigma$ as the tolerance; (4) perform cuts keeping only stars that match the dwarf CMD to within $2 \sigma$; (5) perform an identical set of steps on a background field; (6) weight PM errors of each target star by background field density at that location; (7) perform a cut in projected distance from the dwarf center of $1 r_{h} ;(8)$ ensure that any new members that pass all these cuts were not already rejected in the original spectroscopic campaigns.

\footnotetext{
${ }^{10}$ https://github.com/Johannes-Sahlmann/pygacs
} 
Table 1

Dwarf Galaxy Properties

\begin{tabular}{|c|c|c|c|c|c|c|c|c|c|c|c|}
\hline ID & Name & $l(\mathrm{deg})$ & $b(\mathrm{deg})$ & R.A. (deg) & Decl. (deg) & $D(\mathrm{kpc})$ & $m-M$ & $\mathbf{M}_{V}$ & $r_{h}\left({ }^{\prime}\right)$ & $\mathrm{RV}\left(\mathrm{km} \mathrm{s}^{-1}\right)$ & Notes \\
\hline 1 & Dra2 & 98.3 & 42.9 & 238.2 & 64.6 & 20.0 & $16.9 \pm 0.3$ & -2.9 & 2.7 & $-347.6 \pm 1.8$ & [1]; RV:[2] \\
\hline 2 & Tuc3 & 315.4 & -56.2 & 359.1 & -59.6 & 25.0 & $17.0 \pm 0.2$ & -2.4 & 6.0 & $-102.3 \pm 2.0$ & [3]; RV:[4] \\
\hline 3 & Hyi1 & 304.5 & -16.5 & 37.4 & -79.3 & 27.6 & $17.2 \pm 0.0$ & -4.7 & 7.4 & $80.4 \pm 0.6$ & {$[5]$} \\
\hline 4 & Car3 & 270.0 & -16.8 & 114.6 & -57.9 & 27.8 & $17.2 \pm 0.1$ & -2.4 & 3.8 & $284.6 \pm 3.4$ & [6]; RV:[7] \\
\hline 5 & Ret2 & 265.9 & -49.6 & 53.9 & -54.0 & 30.0 & $17.4 \pm 0.2$ & -2.7 & 3.6 & $62.8 \pm 0.5$ & [8]; RV:[9] \\
\hline 6 & Cet2 & 156.5 & -78.5 & 19.5 & -17.4 & 30.0 & $17.4 \pm 0.2$ & 0.0 & 1.9 & $\ldots$ & [3]; RV:[10] \\
\hline 7 & Tri2 & 140.9 & -23.8 & 33.3 & 36.2 & 30.0 & $17.4 \pm 0.1$ & -1.8 & 3.9 & $-381.7 \pm 1.1$ & [11]; RV:[12] \\
\hline 8 & Car2 & 270.0 & -17.1 & 114.1 & -58.0 & 36.2 & $17.8 \pm 0.1$ & -4.5 & 8.7 & $477.2 \pm 1.2$ & [6]; RV:[7] \\
\hline 9 & Pic2 & 269.6 & -24.1 & 101.2 & -59.9 & 45.0 & $18.3 \pm 0.1$ & -3.2 & 3.6 & $\ldots$ & [13] \\
\hline 10 & Tuc4 & 313.3 & -55.3 & 0.7 & -60.9 & 48.0 & $18.4 \pm 0.2$ & -3.5 & 9.1 & $\ldots$ & {$[3]$} \\
\hline 11 & Gru2 & 351.1 & -51.9 & 331.0 & -46.4 & 53.0 & $18.6 \pm 0.2$ & -3.9 & 6.0 & $\ldots$ & [3] \\
\hline 12 & Tuc5 & 316.3 & -51.9 & 354.3 & -63.3 & 55.0 & $18.7 \pm 0.3$ & -1.6 & 1.0 & $\ldots$ & {$[3 ; 26]$} \\
\hline 13 & Tuc2 & 327.9 & -52.8 & 343.0 & -58.6 & 57.0 & $18.8 \pm 0.2$ & -3.8 & 9.8 & $-129.1 \pm 3.5$ & [8]; RV:[14] \\
\hline 14 & Sag2 & 18.9 & -22.9 & 298.2 & -22.1 & 67.0 & $19.1 \pm 0.1$ & -5.2 & 2.0 & $\ldots$ & {$[11]$} \\
\hline 15 & Lae3 & 63.6 & -21.2 & 316.8 & 15.0 & 67.0 & $19.1 \pm 0.1$ & -4.4 & 0.4 & $\ldots$ & {$[1]$} \\
\hline 16 & Hor2 & 262.5 & -54.1 & 49.1 & -50.0 & 78.0 & $19.5 \pm 0.2$ & -2.6 & 2.1 & $\ldots$ & [15] \\
\hline 17 & Hor1 & 270.9 & -54.7 & 43.9 & -54.1 & 79.0 & $19.5 \pm 0.2$ & -3.4 & 1.3 & $112.8 \pm 2.6$ & [8]; RV:[16] \\
\hline 18 & $\mathrm{Phx} 2$ & 323.3 & -60.2 & 355.0 & -54.4 & 83.0 & $19.6 \pm 0.2$ & -2.8 & 1.1 & $\ldots$ & [8] \\
\hline 19 & Eri3 & 274.3 & -59.6 & 35.7 & -52.3 & 87.0 & $19.7 \pm 0.2$ & -2.0 & 0.5 & $\ldots$ & {$[8 ; 26]$} \\
\hline 20 & Vir1 & 276.9 & 59.6 & 180.0 & -0.7 & 91.0 & $19.8 \pm 0.2$ & -0.3 & 1.8 & $\ldots$ & {$[17]$} \\
\hline 21 & Ret3 & 273.9 & -45.6 & 56.4 & -60.4 & 92.0 & $19.8 \pm 0.3$ & -3.3 & 2.4 & $\ldots$ & {$[3]$} \\
\hline 22 & Ind 1 & 347.3 & -42.6 & 317.2 & -51.2 & 100.0 & $20.0 \pm 0.2$ & -3.5 & 1.3 & $\ldots$ & [8] \\
\hline 23 & Aqu2 & 55.1 & -53.0 & 338.5 & -9.3 & 107.9 & $20.2 \pm 0.1$ & -4.4 & 5.1 & -71.1 & [18]; RV:[18] \\
\hline 24 & Pic1 & 257.1 & -40.4 & 70.9 & -50.3 & 114.0 & $20.3 \pm 0.2$ & -3.1 & 0.9 & $\ldots$ & {$[8]$} \\
\hline 25 & $\mathrm{Cra} 2$ & 282.9 & 42.0 & 177.3 & -18.4 & 117.5 & $20.4 \pm 0.0$ & -8.2 & 31.2 & $87.5 \pm 0.4$ & [19]; RV:[20] \\
\hline 26 & Gru1 & 338.6 & -58.8 & 344.2 & -50.2 & 120.0 & $20.4 \pm 0.2$ & -3.4 & 1.8 & $-140.5 \pm 2.4$ & [8]; RV:[14] \\
\hline 27 & Hya2 & 295.6 & 30.5 & 185.4 & -32.0 & 134.0 & $20.6 \pm 0.2$ & -4.8 & 1.7 & $303.1 \pm 1.4$ & [21]; RV:[22] \\
\hline 28 & Col1 & 231.6 & -28.9 & 82.9 & -28.0 & 182.0 & $21.3 \pm 0.2$ & -4.5 & 1.9 & $\ldots$ & {$[3]$} \\
\hline 29 & Ind2 & 354.0 & -37.4 & 309.7 & -46.2 & 214.0 & $21.6 \pm 0.2$ & -4.3 & 2.9 & $\ldots$ & {$[3]$} \\
\hline 30 & Peg3 & 69.8 & -41.8 & 336.1 & 5.4 & 215.0 & $21.7 \pm 0.1$ & -3.4 & 0.8 & $-222.9 \pm 2.6$ & [23]; RV:[24] \\
\hline 31 & Cet3 & 163.8 & -61.1 & 31.3 & -4.3 & 251.0 & $22.0 \pm 0.2$ & -2.5 & 1.2 & $\ldots$ & {$[17]$} \\
\hline 32 & Eri2 & 249.4 & -51.4 & 56.1 & -43.5 & 380.0 & $22.9 \pm 0.2$ & -6.6 & 1.5 & $75.6 \pm 2.4$ & [8]; RV:[25] \\
\hline
\end{tabular}

Note. Properties of the dwarf galaxies that are the subject of this study listed in order of increasing distance. Column 1 lists an ID, followed by our naming convention, galactic longitude and latitude $(l, b)$, R.A. and decl., heliocentric distance, distance modulus, absolute magnitude, half-light radius, and radial velocity (if measured). Citations are given in the notes, and refer to [1] Laevens et al. (2015a), [2] Martin et al. (2016), [3] Drlica-Wagner et al. (2015), [4] Simon et al. (2017), [5] Koposov et al. (2018), [6] Torrealba et al. (2018), [7] Li et al. (2017), [8] Koposov et al. (2015a), [9] Simon et al. (2015), [10] Conn et al. (2018a), [11] Laevens et al. (2015b), [12] Kirby et al. (2017), [13] Drlica-Wagner et al. (2016), [14] Walker et al. (2016), [15] Kim \& Jerjen (2015), [16] Koposov et al. (2015b), [17] Homma et al. (2018), [18] Torrealba et al. (2016a), [19] Torrealba et al. (2016b), [20] Caldwell et al. (2017), [21] Martin et al. (2015), [22] Kirby et al. (2015), [23] Kim et al. (2015), [24] Kim et al. (2016), [25] Li et al. (2018), and [26] Conn et al. (2018b).

In Figures 1-3, we show three illustrative examples of this process. In the figures, we show a CMD of the spectroscopic members (green plus signs), the new members (yellow squares), the control field stars (red diamonds), and the candidate members rejected by the projected distance cut (blue circles). In Figure 1, we show the case for Car2 in which we successfully add 14 new members to the spectroscopic sample thereby decreasing the final errors by a factor of 2 . In Figure 2, we show the case for Hyil, in which we add eight new members to the spectroscopic sample of 30 from Koposov et al. (2018). Four of these eight new member stars are quite bright, and therefore constraining, as can be seen in the figure. Finally, in Figure 3, we show a third example of a dwarf (Hor1) where we do not add that many new members, but where the result is still interesting given how sparse the current sample is.

In the vast majority, we add only between three and five stars, but some of these are bright and therefore useful additions to these sparse samples, affording modest improvements of the PM errors. However, in many fields, we add mostly faint stars, on the limit of what Gaia can do in DR2. A good example is Cra2, a field in which we add many stars (59), but the majority are very faint such that the resultant errors are barely an improvement over the spectroscopic-only sample PMs. For this field, we impose a magnitude cut at $G>20$. For all other fields, we do not employ this cut so as to recover the faint spectroscopic sample.

Table 2 presents our measurements, including how many stars are added for each field. Most values are consistent within the errors of the spectroscopic samples presented in Fritz et al. (2018) and Simon (2018). Exceptions to this consistency include our value for Tuc2, which differs at the $3 \sigma$ level in $\delta$ from Fritz et al. (2018) and at the $2.5 \sigma$ level also in $\delta$ from Simon (2018), but is very consistent in $\alpha$. We do consider and attempt to measure PMs for Peg3 and Eri2, both of which have measured RV's. However, for Peg3, Fritz et al. (2018) find no spectroscopic matches, and for Eri2, which is at $380 \mathrm{kpc}$, our pipeline to find additional members only produces two additional stars. The resulting PM errors are still in the $\sim 0.3 \mathrm{mas} \mathrm{yr}^{-1}$ range, which at that distance corresponds to $\sim 540 \mathrm{~km} \mathrm{~s}^{-1}$, hopelessly large. This large error, in conjunction with the fact that membership can be ruled out via its location 

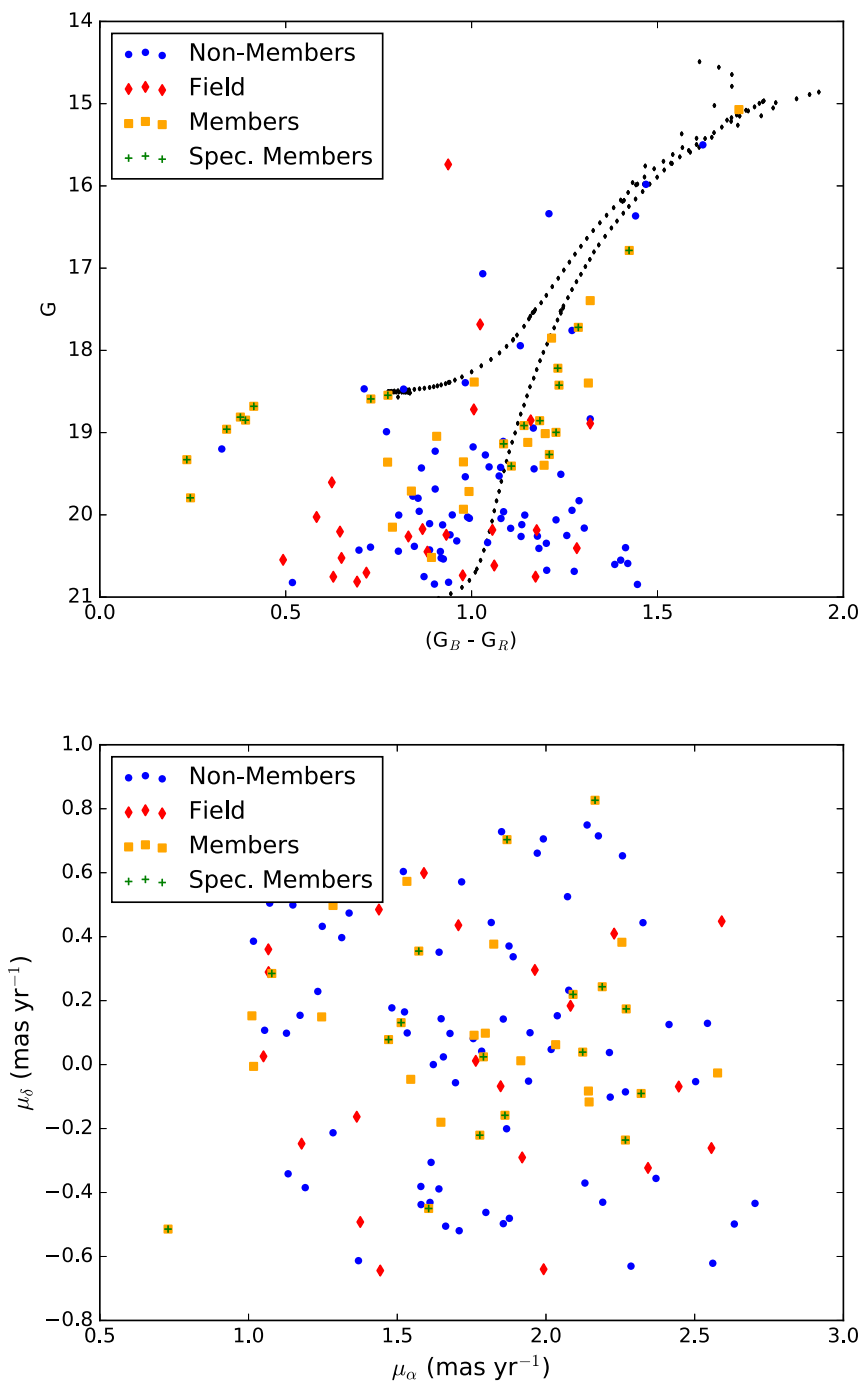

Figure 1. Top: our selection process for Car2. Green plus signs show the spectroscopic member stars that make it through our quality cuts; yellow, blue, and red stars are consistent with the spectroscopic PMs. Red stars are from our control "background" field, blue stars are target members that get cut due to our $1-r_{h}$ radius selection, and yellow stars (without plus signs overlaid) are 14 newly added members, used in addition to the spectroscopic members for the PM calculation. A PARSEC isochrone is overlaid for illustrative purposes. Bottom: the PM field, with the same color scheme.

and distance alone (see Section 3), prompt us to eliminate it from further analysis.

Soon after submission of this work, Massari \& Helmi (2018) presented a paper searching for additional photometric members for seven dwarfs in DR2, with two dwarfs overlapping with our sample, Car2 and Ret2. Their method is not dissimilar to ours. For these two dwarfs, they also choose additional members based on an initial RV guess, which is refined iteratively by $2.5 \sigma$ cuts in PM and parallax, followed by a further culling on the basis of a CMD, and projected distance from the dwarf center. Our method also utilizes PM, CMD, and projected distance cuts (though there are differences in the choice of tolerance-we choose $2 \sigma$ for our PM and CMD cuts). A difference is that we do not further iterate on the selection, as we found that this mostly added faint stars, while they do not apply their selection on a control field, i.e., weight their results by a control field. Nonetheless, our results are very consistent with theirs. The reason that they report more new candidate
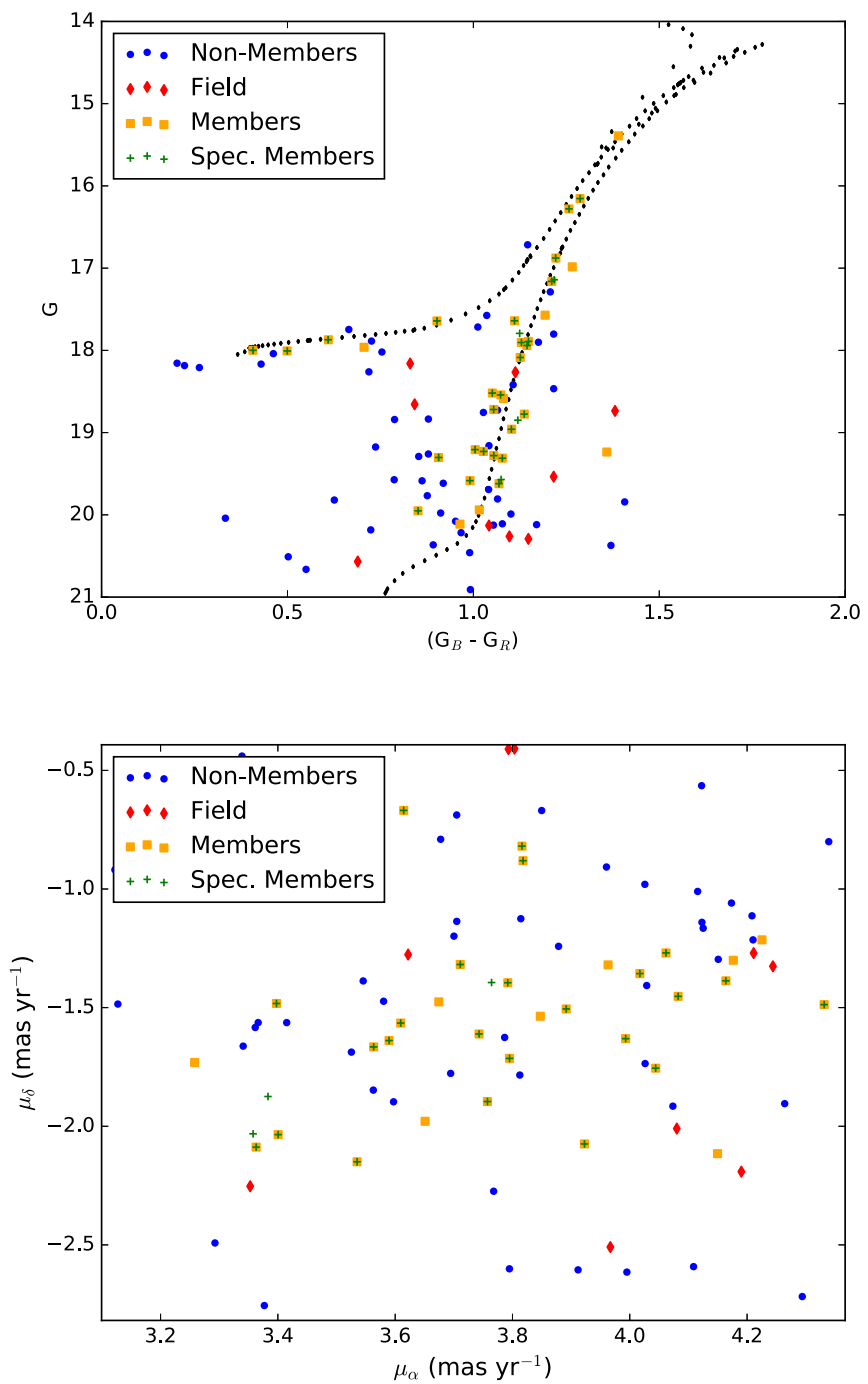

Figure 2. Top: same as Figure 1, but for Hyil. In this case, we add eight new members to the 30 spectroscopic members, some of which are relatively bright. Note that 4 of the 30 spectroscopic members do not pass our parallax quality cuts and are therefore not used in our analysis, but we show them here (green plus signs) for completeness.

members than we do is because of the very strict cut $\left(1-r_{h}\right)$ that we make in projected distance from the dwarf center. Massari \& Helmi do not report what projected distance cuts they use, but if we do not make this cut then we obtain similar numbers of candidate members as they do: 47 total members for Ret2 and 61 for Car 2. The resulting errors from using this larger sample of candidate members are also consistent with their errors, and are slightly larger than those presented here with the $1 r_{\mathrm{h}}$ cut. This is consistent with our finding that adding many additional photometric members, that are mostly faint, does not improve the precision. This explains why we concentrated on searching for bright members very close to the UFD core. We do note, however, that the Massari \& Helmi method will be helpful to train further RV studies of these dwarfs and to better understand their structural properties.

Gaia Collaboration et al. (2018b) have presented DR2 PMs for nine classical dwarf spheroidals of the MW. Even though the regime, in terms of number of stars, is rather different than for the UFDs presented here, we mention their work here in order to provide a general consistency check of Gaia PMs. Specifically, they are able to compare their results to previous 

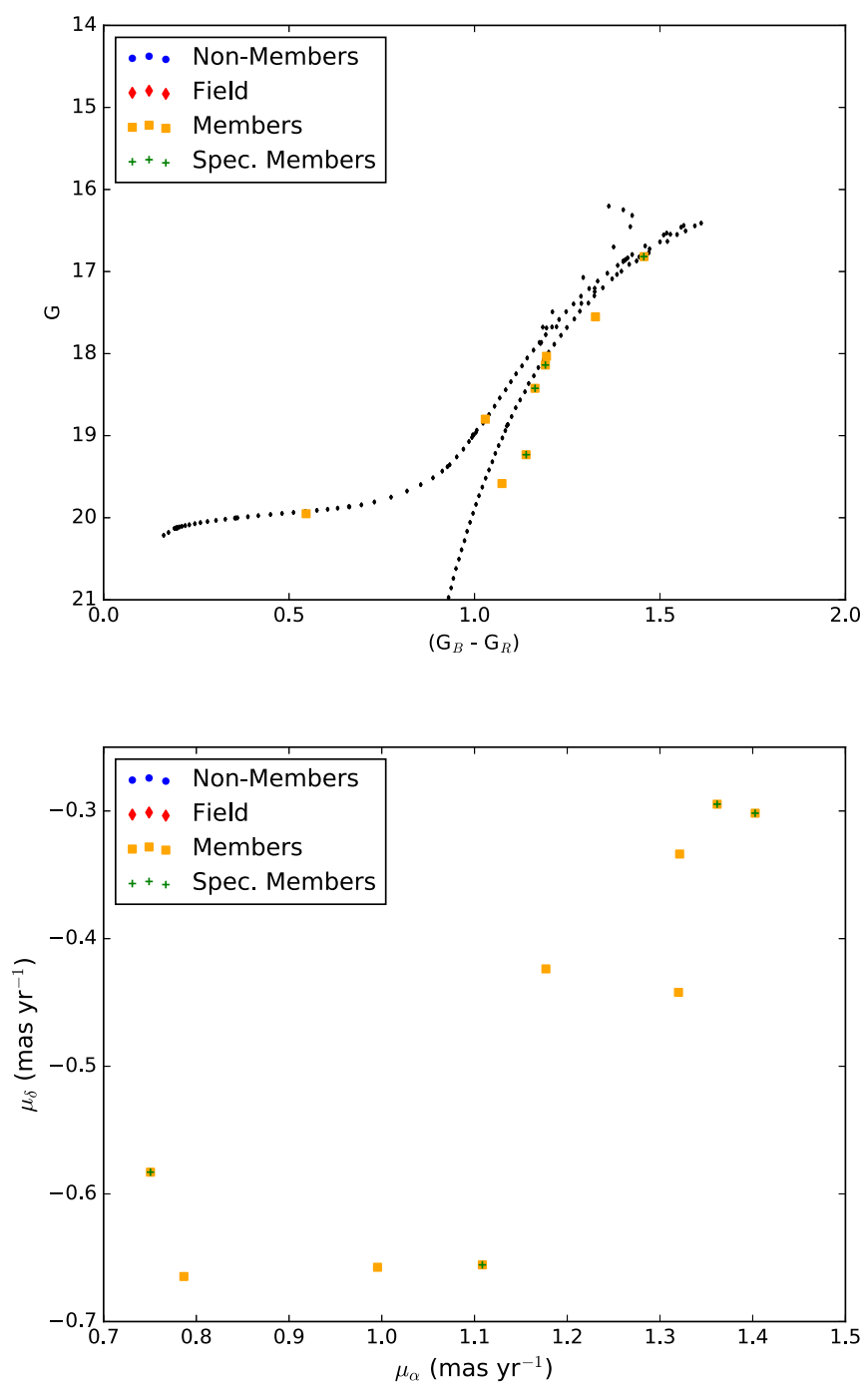

Figure 3. Same as Figures 1 and 2, but for the more modest gains in the case of Hor1. We are, however, adding relatively bright (and therefore relatively wellmeasured) stars.

Table 2

PM Measurements

\begin{tabular}{lrrcccc}
\hline \hline Name & Nspec & Nnew & $\begin{array}{c}\mu_{\alpha^{*}} \\
\left(\mathrm{mas} \mathrm{yr}^{-1}\right)\end{array}$ & $\begin{array}{c}\delta_{\mu \alpha^{*}} \\
\left(\mathrm{mas} \mathrm{yr}^{-1}\right)\end{array}$ & $\begin{array}{c}\mu_{\delta} \\
\left(\mathrm{mas} \mathrm{yr}^{-1}\right)\end{array}$ & $\begin{array}{c}\delta_{\mu \delta} \\
\left(\mathrm{mas} \mathrm{yr}^{-1}\right)\end{array}$ \\
\hline Dra2 & 6 & 4 & 1.165 & 0.260 & 0.866 & 0.270 \\
Tuc3 & 20 & 12 & -0.026 & 0.037 & -1.679 & 0.039 \\
Hyi1 & 26 & 8 & 3.773 & 0.032 & -1.581 & 0.030 \\
Car3 & 3 & 1 & 3.065 & 0.095 & 1.567 & 0.104 \\
Ret2 & 22 & 3 & 2.398 & 0.039 & -1.319 & 0.048 \\
Tri2 & 3 & 0 & 0.588 & 0.187 & 0.554 & 0.161 \\
Car2 & 15 & 14 & 1.802 & 0.038 & 0.084 & 0.038 \\
Tuc2 & 16 & 8 & 0.966 & 0.049 & -1.380 & 0.062 \\
Hor1 & 4 & 5 & 0.926 & 0.070 & -0.569 & 0.065 \\
Aqu2 & 2 & 3 & -0.491 & 0.306 & -0.049 & 0.266 \\
Cra2 & 51 & 59 & -0.246 & 0.052 & -0.227 & 0.026 \\
Gru1 & 4 & 2 & -0.254 & 0.220 & -0.532 & 0.288 \\
Hya2 & 5 & 6 & -0.417 & 0.402 & 0.179 & 0.339 \\
\hline
\end{tabular}

Note. Our measured PMs. Column 1 lists the name, followed by the number of stars from the spectroscopic sample of Fritz et al. (2018) that pass our cuts, the number of new members that we add, and the resulting PM values in $\mu_{\alpha \star}, \delta \mu_{\alpha \star}$, $\mu_{\delta}$, and $\delta \mu_{\delta}$. studies using independent instruments/methods (both groundbased and with HST), and find that, in general, their Gaia PMs are roughly consistent with previous determinations.

Galactocentric quantities are calculated using the same Cartesian coordinate system $(X, Y, Z)$ as in Kallivayalil et al. (2013; see also Sohn et al. 2012, 2013), and are mimicked when making predictions from the Aquarius simulation. In this system, the origin is at the Galactic center, the $X$-axis points in the direction from the Sun to the Galactic center, the $Y$-axis points in the direction of the Sun's Galactic rotation, and the $Z$-axis points toward the Galactic north pole. The position and velocity of the dwarfs in this frame can be derived from the observed sky positions, distances, line-of-sight velocities, and PMs. Errors in the Galactocentric quantities are calculated by doing 100,000 Monte Carlo drawings over the errors in the measured PMs, radial velocities and distance moduli. Solar parameters are from Bovy et al. (2012).

\section{Results}

\subsection{Simulation Setup}

We use the Aquarius simulations (Springel et al. 2008) to identify LMC analogs within the cosmological scenario of $\Lambda \mathrm{CDM}$. The Aquarius halos consist of six zoom-in DM-only cosmological simulations of MW-sized halos, with virial masses in the range $(0.8-1.8) \times 10^{12} M_{\odot}$. The closest LMC analog, the LMCa, was chosen to match the present-day position and measured orbital velocity of the LMC (Kallivayalil et al. 2006b), and has a virial mass of $M_{200}=3.6 \times$ $10^{10} M_{\odot}$, corresponding to a circular velocity of $65 \mathrm{~km} \mathrm{~s}^{-1}$. The corresponding host halo has a virial mass of $M_{200}=1.8 \times$ $10^{12} M_{\odot}$ at $z=0$, on the upper end of current MW mass estimates (Bland-Hawthorn \& Gerhard 2016), but consistent with early analyses of Gaia DR2 data (Fritz et al. 2018; Simon 2018; Watkins et al. 2018). Since this is a fully cosmological simulation, we know the full orbital history of LMCa as well as that of all DM particles that are bound to it pre-infall. The distance and velocity of LMCa most closely match those of the real LMC when it is at first pericenter (which in the simulation occurs at $t=9.6 \mathrm{Gyr}$ ), but is still consistent with measurements during a second pericenter passage at $t=13.3 \mathrm{Gyr}$. We therefore consider mostly here the scenario of first infall, but discuss the implications for the case of a second passage as well. The MW analog has $M_{200}=1.4$ and $1.6 \times 10^{12} M_{\odot}$ at the times of these first and second pericenter passages. More details on the identification of our LMCa can be found in S17 and Sales et al. (2011).

We follow the evolution of LMCa using the level 3 of the Aquarius halo A (Aq-A-3) with a mass and space resolution $4.9 \times 10^{4} M_{\odot}$ and $120 \mathrm{pc}$ respectively. Using the merger trees we trace backwards in time the LMCa orbital evolution and identify all particles that were initially bound to this structure before its infall onto the host halo. All particles associated to the LMCa before infall (defined as the last snapshot where the LMCa was the central of its own friends-of-friends group) provide a fair sampling of the phase space properties expected of any material initially associated to the LMC. In particular, since subhalos roughly follow the DM outside the first inner kiloparsec of halos, looking at the distribution of the tagged particles at the time of first or second pericenter passage provide useful predictions for the present-day positions and 

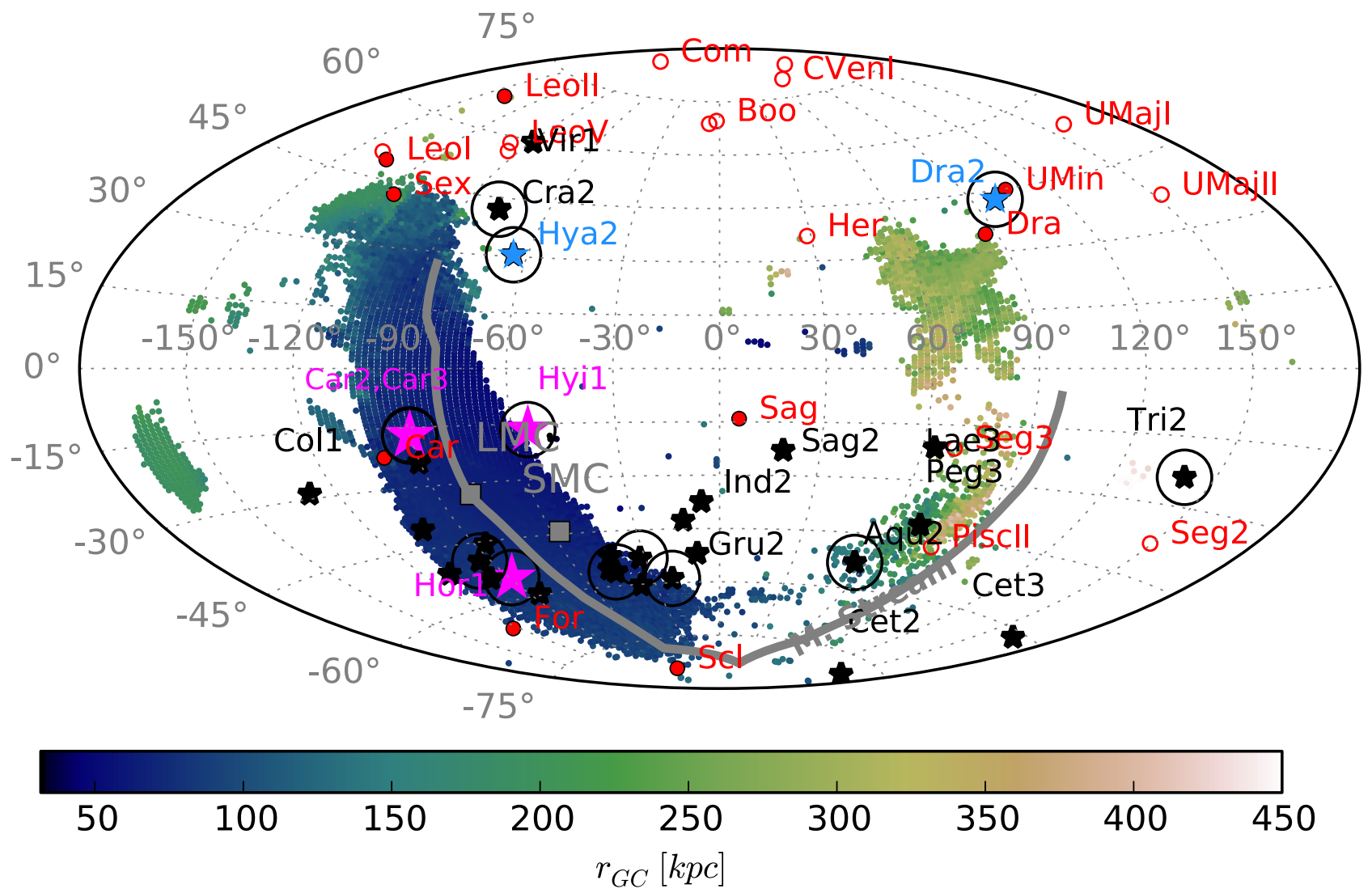

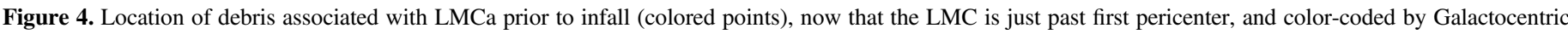

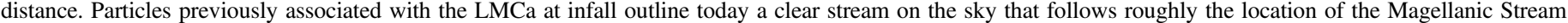

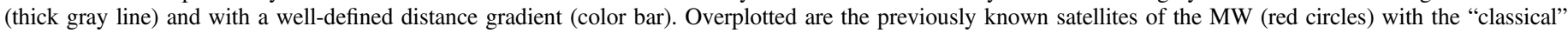

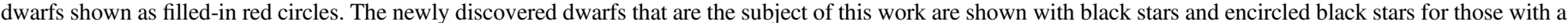

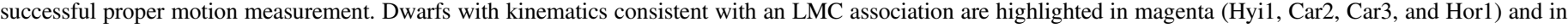
light blue we show Hya2 and Dra2 for which membership certainty deserves more analysis in the future. See the text and Figures 5 and 6 for more details.

velocities to be expected of any dwarf companion that was brought onto the MW as part of the LMC group (see S17 for a more detailed discussion).

Our approach relies on two assumptions: (i) baryons will not statistically bias the orbital properties of LMC analogs in a sample of MW-like hosts, and (ii) satellites of the LMC follow the same radial distribution as that of its DM halo before infall into the MW. Regarding the first point, we note that Patel et al. (2017) have shown that LMC analogs in Illustris 1 (with baryons) versus with Illustris Dark 1 (only DM) do not exhibit markedly different orbital properties, providing further validation to our analysis. The second point deserves more caution as baryonic processes have been shown in some cases to preferentially disrupt inner satellites due to the increased tides associated with the disks (e.g., D’Onghia et al. 2010; Ahmed et al. 2017; Garrison-Kimmel et al. 2017). Encouragingly, these effects are presumably smaller for a less massive disk such as the LMC and are increasingly weaker with distance to the host. Although this is not well known in dwarf galaxies, for more massive systems the radial distribution of luminous satellites is expected to show little systematic difference with the DM beyond $\sim 10 \%$ of the virial radius of the host (Sales et al. 2007; Vogelsberger et al. 2014). Assuming a virial radius of $130 \mathrm{kpc}$ for the LMC before infall, it would limit the applicability of our results for dwarfs closer to the LMC than $13 \mathrm{kpc}$ (see S17). All dwarfs explored in this paper, and in particular those considered likely members of the LMC group, lie well beyond this limit, with the closest being Car2 at $\sim 25 \mathrm{kpc}$.

\subsection{Comparison to LMC Debris}

At the time of first pericenter passage for the LMCa, some tidal disruption due to the host halo has already set in and has started partially unbinding the group. These unbound particles, however, follow a very well-defined pattern on the sky, distance, and velocity space due to the common orbital properties with the LMCa that can be compared to observations to determine which of the new dwarfs match these predictions. Figure 4 shows the footprint of the LMCa debris on the sky at the time of first pericenter. The DM particles lie along a welldefined tidal tail that is roughly coincident with the real Magellanic Stream, sketched in with a thick gray line (Nidever et al. 2010). Overplotted are the Galactic coordinates of the 32 new candidate dwarfs (star symbols). The spatial distribution of the new dwarfs coincides quite well with the sky distribution of DM particles that are (or were) bound to the LMC.

The DM particles are color-coded by Galactocentric distance. There is a clear gradient in distance along the Stream. LMC debris can be distributed as far out as $300 \mathrm{kpc}$ even at first pericenter. Nonetheless, because LMC debris still must lie close to the orbital plane of the LMC, Figure 4 shows that sky distribution and distance by themselves are quite good determinants of LMC membership. We find that no particles in our LMC analog cover the region of sky inhabited by Eri2, Ind2, Cet2, Tri2, Cet3, and Vir1. In these cases, the probability of association is formally zero in the simulation, and the $\mathrm{LMCa}$ stream does not predict any dwarf/particle in those regions. 
Table 3

Predicted PMs and Radial Velocities

\begin{tabular}{|c|c|c|c|c|c|c|}
\hline Name & 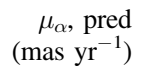 & $\begin{array}{r}\mu_{\delta}, \text { pred } \\
\left(\operatorname{mas~yr}^{-1}\right)\end{array}$ & $\begin{array}{r}\mathrm{RV}_{\text {pred }} \\
\left(\mathrm{km} \mathrm{s}^{-1}\right)\end{array}$ & Nstars & 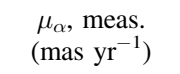 & 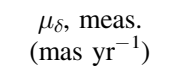 \\
\hline Pic2 & $1.69_{-0.04}^{+0.05}$ & $0.77_{-0.15}^{+0.13}$ & $333.83_{-38.78}^{+42.84}$ & 2 & $\ldots$ & $\ldots$ \\
\hline Tuc4 & $1.26_{-0.15}^{+0.17}$ & $-2.19_{-0.0}^{+0.02}$ & $33.51_{-11.07}^{+14.66}$ & 0 & $\ldots$ & $\ldots$ \\
\hline Gru2 & $0.26_{-0.16}^{+0.08}$ & $-2.24_{-0.02}^{+0.08}$ & $-132.32_{-2.23}^{+17.27}$ & 2 & $\ldots$ & $\ldots$ \\
\hline Tuc5 & $0.91_{-0.14}^{+0.12}$ & $-1.94_{-0.02}^{+0.01}$ & $33.49_{-10.64}^{+8.19}$ & 0 & $\cdots$ & $\cdots$ \\
\hline Sag2 & $-0.01_{-0.03}^{+0.03}$ & $-1.3_{-0.04}^{+0.02}$ & $-339.54_{-11.08}^{+6.35}$ & 0 & $\cdots$ & $\cdots$ \\
\hline Lae3 & $0.07_{-0.06}^{+0.08}$ & $-0.92_{-0.01}^{+0.01}$ & $-477.73_{-13.26}^{+3.28}$ & 0 & $\ldots$ & $\cdots$ \\
\hline Hor2 & $1.25_{-0.05}^{+0.06}$ & $-0.65_{-0.03}^{+0.03}$ & $159.81_{-30.68}^{+26.76}$ & 0 & $\ldots$ & $\ldots$ \\
\hline Phx2 & $0.67_{-0.08}^{+0.03}$ & $-1.25_{-0.01}^{+0.01}$ & $-15.45_{-10.48}^{+5.22}$ & 4 & $-0.54 \pm 0.10$ & $-1.17 \pm 0.12$ \\
\hline Eri3 & $1.04_{-0.07}^{+0.06}$ & $-0.75_{-0.02}^{+0.0}$ & $126.18_{-27.29}^{+29.89}$ & 1 & $\ldots$ & $\ldots$ \\
\hline Ret3 & $1.12_{-0.05}^{+0.05}$ & $-0.32_{-0.04}^{+0.03}$ & $229.85_{-32.02}^{+31.41}$ & 2 & $\ldots$ & $\ldots$ \\
\hline Ind 1 & $0.26_{-0.01}^{+0.01}$ & $-0.98_{-0.01}^{+0.01}$ & $-93.93_{-1.28}^{+1.88}$ & 4 & $-0.23 \pm 0.15$ & $-1.22 \pm 0.15$ \\
\hline Pic1 & $0.77_{-0.02}^{+0.01}$ & $-0.1_{-0.01}^{+0.08}$ & $176.68_{-20.77}^{+16.89}$ & 3 & $-0.08 \pm 0.24$ & $0.07 \pm 0.31$ \\
\hline Col1 & $0.16_{-0.0}^{+0.01}$ & $-0.1_{-0.0}^{+0.0}$ & $340.17_{-13.5}^{+18.71}$ & 4 & $-0.42 \pm 0.14$ & $-0.15 \pm 0.19$ \\
\hline Peg3 & $0.12_{-0.02}^{+0.02}$ & $-0.27_{-0.01}^{+0.0}$ & $-327.12_{-0.97}^{+8.9}$ & 2 & $\ldots$ & $\ldots$ \\
\hline
\end{tabular}

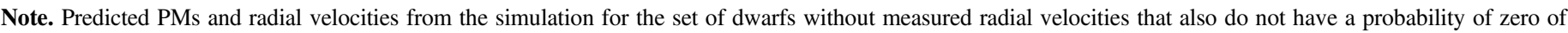

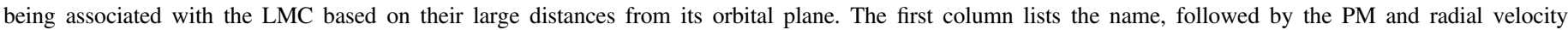

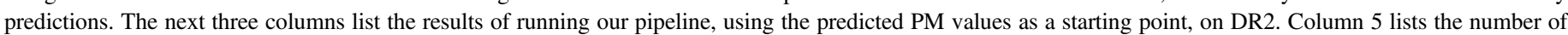
stars found to be consistent with this prediction, and if more than 3 , then their weighted-average PMs.

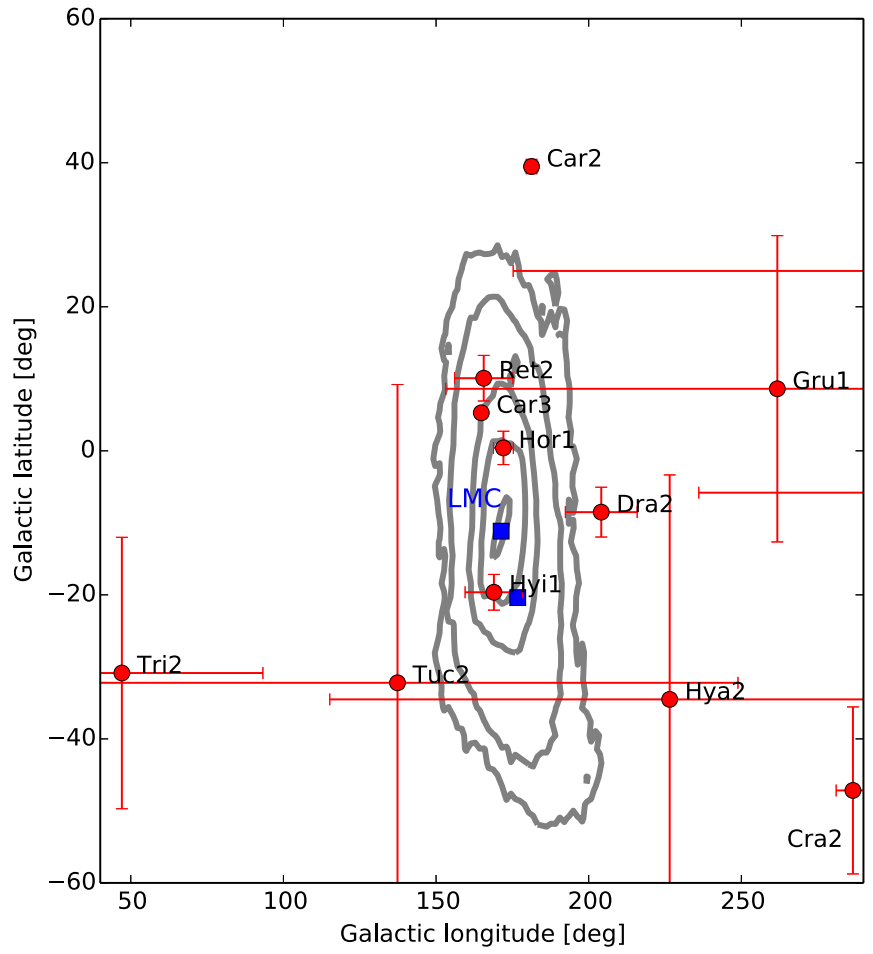

Figure 5. Direction of the axis of orbital angular momentum of LMCa debris (gray density contours enclose from the innermost to the outermost lines $5 \%-95 \%$ of all LMCa particles, respectively) along with those measured for dwarfs in this study that lie close to this direction. Hor1, Hyi1, Car3, and Ret2 are clearly coincident with the Magellanic system, a condition necessary but not sufficient for a common origin with the LMC. Dra2, Tuc2, Hya2, and Gru1 are marginally consistent given their measurement errors.

Certainty of membership for the remaining dwarfs, however, comes from adding the measured 3D velocities (radial velocities plus PMs) to the sky positions and distances, to get full $6 \mathrm{D}$ quantities, and from comparing these $6 \mathrm{D}$ quantities to the simulation predictions. We first compare the orbital poles of the satellites with 3D measurements to the orbital pole of the LMCa system. Figure 5 plots the galactic $l$ and $b$ positions of the orbital poles, which are preserved and should be consistent for infalling groups, for LMCa particles (gray density contours) and for the subset of measured dwarfs that also inhabit this region of angular momentum space. Hor1, Hyi1, Car3, and Ret2 are clearly consistent with the angular momentum direction of the LMC system, listed in descending order of significance as represented by the density of LMCa debris in the same region. Dra2, Tuc2, Hya2, and Gru1 are also consistent within their measured $1 \sigma$ errors, where the errors in the poles are calculated as standard deviation of 1000 Monte Carlo drawings over the measurement errors in Galactocentric $X, Y, Z$ and $V_{X}, V_{Y}$ and $V_{Z}$.

We next consider the Galactocentric radial and tangential velocities of the debris, and compare to those measured for the dwarfs. Following S17, we select all DM particles once part of the LMCa system that lie within a $5^{\circ}$ radius of the sky positions of the dwarfs. We check their Galactocentric distances, and their Galactocentric $V_{X}, V_{Y}$ and $V_{Z}$ values. We then plot, in Figure 6, the corresponding observed distance of the dwarf $\pm 20 \%$ range (red regions) and the observed velocities and errors. The radius and distance tolerance were chosen to maximize the total number of potential LMCa particles in a given region of sky, while still giving peaked (confined) predicted velocity histograms.

At least four dwarfs show clearly consistent positions and velocity measurements compared to our predictions: Hyil, Car3, Car2, and Hor1 (red labels), presenting a compelling case of probable membership to the LMC group. Ret2, Tuc2, and Gru1 have velocity components that are not consistent within $3 \sigma$ of our predictions and are unlikely members of the LMC group according to our analysis. Notice that Hya2 is an interesting case. In a first pericenter passage scenario, Hya2 occupies the foremost tip of the leading arm of the stream. Only a few particles are expected in that area of the sky (see Figure 4), but despite this seemingly low chance of association, their velocities agree well with the observed ones for Hya2, 


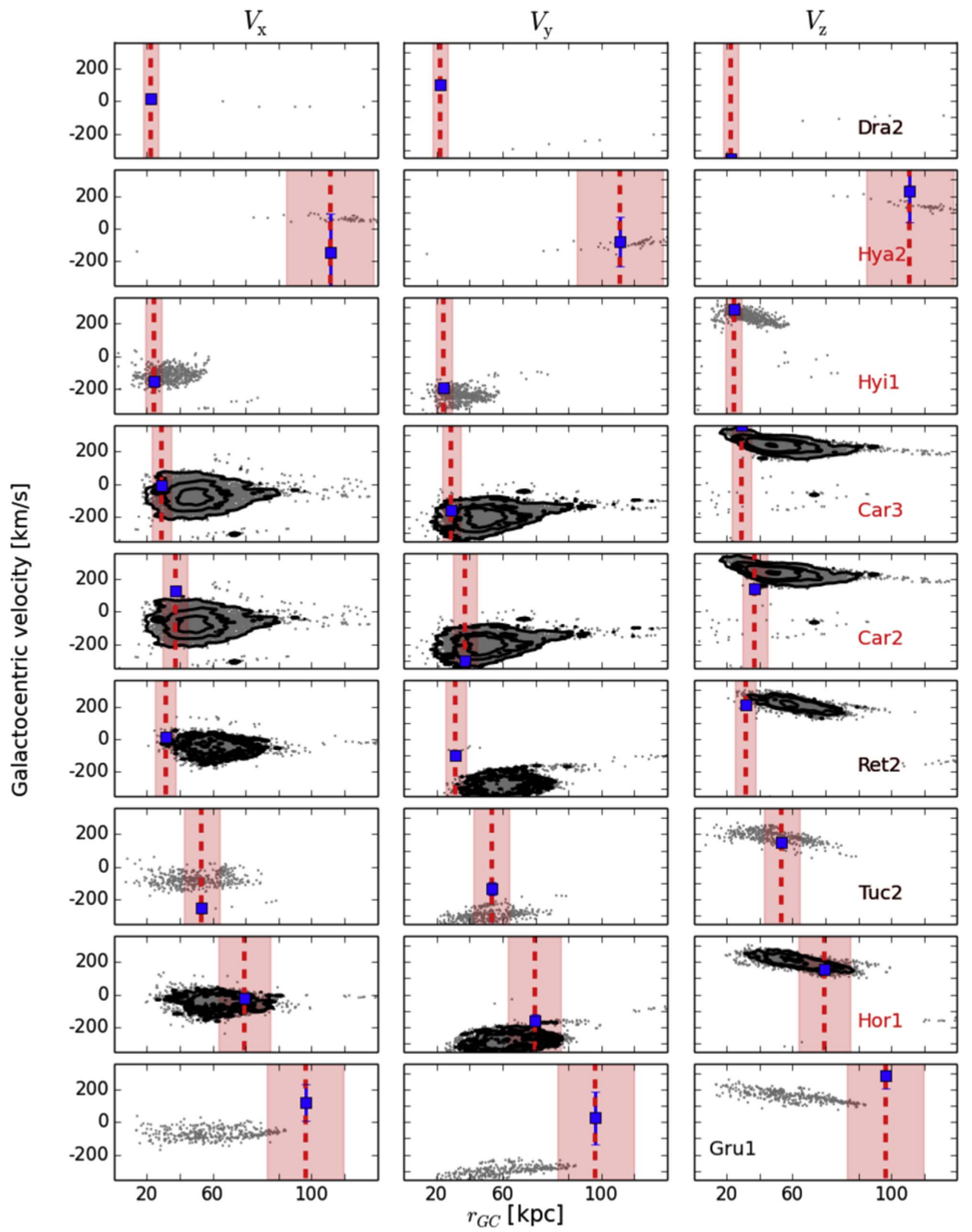

Figure 6. Galactocentric velocity in $V_{X}, V_{Y}$, and $V_{Z}\left(\mathrm{~km} \mathrm{~s}^{-1}\right)$ vs. Galactocentric distance (kpc) for the observed dwarfs vs. LMCa debris from the simulation, for nine dwarfs of interest (labeled in each right-hand side panel) sorted by galactic latitude. Gray dots represent the LMCa DMz particles located within 5 deg $^{2}$ of the position of the dwarf. Red dashed lines correspond to the observed radial distance of each dwarf together with the $\pm 20 \%$ range used to average the predictions in Table 3 . The observed velocities are indicated with blue square symbols. Hyi1, Car3, Car2, and Hor1 are likely associated to the LMC (red labels) whereas Gru1, Tuc2, and Ret2 are currently disfavored. Hya2 and Dra2 cannot be ruled out and deserved further analysis (see the text for more details). 

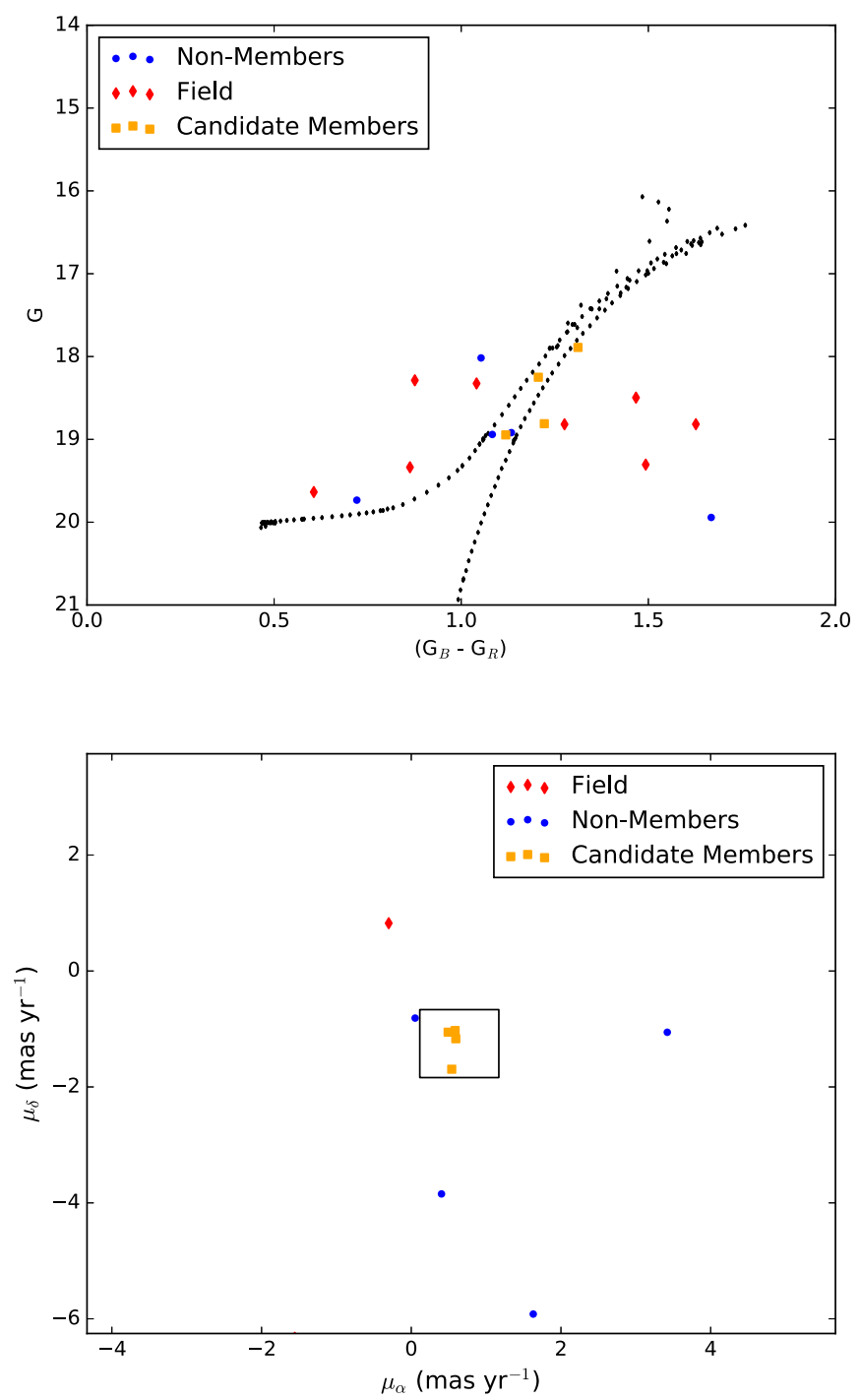

Figure 7. Same as Figures 1 and 2, but for the prediction of $\mathrm{Phx} 2$. Blue points are candidate member stars, while red points show our control (background) field.

suggesting that association of Hya2 to the LMC might not be quickly ruled out. On the other hand, though Dra2 is consistent within $1 \sigma$ with the position of the orbital pole (see Figure 5), it has a location on the sky that is disfavored and we find almost no particles associated with the stream (however, see the discussion in Section 3.3). For completeness, we include this case in the first row of Figure 6 for easy comparison to the other cases.

\subsection{Association with the LMC in the Case of Second Pericenter Passage}

A similar analysis as that presented in the previous section can be done for the case of LMCa transiting through its second pericenter passage. We have checked that our results presented in Figures 4 through 6 are still valid even if a second approach is considered. Moreover, because of the more extended footprint of the stream over the North Hemisphere due to the completion of one full orbit for the LMC (see Figure 1 in Sales et al. 2011), the chances of association of Hya2 are significantly improved.

It is worth mentioning that the dispersion of the stream on the sky during the second passage also allows for a better consideration of the case for Dra2. We find that for that region of the sky, the LMCa debris predicts velocities consistent with those measured for Dra2. This might imply that had the LMCa been more massive (and therefore extended) the sky position of Dra2 could have been more sampled even in first pericenter passage. This does not remove the fact that such large separation from the main stream is less likely to represent a previous association to the LMC. Based on our results, we cannot rule out the association of Dra2, an issue that deserves further examination in the future.

\subsection{Predictions for Galaxies without Radial Velocity Measurements}

We now use the velocity information from the simulation to ascertain whether any of the galaxies in Table 1 without RV measurements might be associated with the Clouds. We convert the Galactocentric velocities measured in the simulation, along with the $25 \%$ and $75 \%$ bounds, for each of these dwarfs to observed parameters ( $\mu_{\alpha \star}, \mu_{\delta}$ and radial velocity). Using these predicted PMs and a generous window around them as the starting point (in lieu of the PM of the spectroscopic sample), we then run our exact same pipeline as in Section 2. If we find more than three stars that pass all of our cuts, we report their "measured" PMs in Table 3. For the case of $\mathrm{Phx} 2$, we find a pretty convincing clump of stars that are consistent with its PM prediction, see Figure 7. This would be an interesting target for radial velocity follow-up.

\section{Conclusions}

We use an LMC analog in the Aquarius simulation to test whether any of the 32 newly discovered ultra-faint dwarf galaxies and candidate dwarf galaxies have 3D velocities, positions, and distances consistent with having come in with that system. The missing pieces of the puzzle were the PMs, which we measure here for 13 UFDs using Gaia DR2.

Starting with the PM of the spectroscopic members, we attempt to identify additional member stars using a series of nested PM and CMD-space cuts. This approach works especially well for Hyi1 and Car2, with more modest gains for other systems.

Using the resulting $6 \mathrm{D}$ velocities, we find that four of these galaxies (Hor1, Car2, Car3, and Hyi1) have come in with the LMC. Ret2, Gru1, and Tuc2 do not match LMC debris within $3 \sigma$ in all their velocity components and so are not as favorable. We rule out Tuc3, Cra2, Tri2, and Aqu2 as potential members.

Hya 2 and Dra2 are interesting cases. Their orbital poles match well with the predictions from the simulation. In the case of Hya2, it occupies the foremost tip of the leading arm of the stream, where only a few particles are expected, but their velocities agree well with those observed. In the case of Dra2, we find almost no particles at its location in the simulation. However, had LMCa been more massive and extended, this area could be more populated with LMC debris even on a first passage, and therefore we cannot rule out the association of Dra2.

Of the dwarfs without measured PMs, five are deemed unlikely on the basis of their positions and distances alone (Eri2, Ind2, Cet2, Cet3, and Vir1). For the remaining sample, we use the simulation to predict PMs and radial velocities, finding that Phx2 has an overdensity of stars in DR2 consistent 
with this PM prediction. It would be a good candidate for radial velocity follow-up.

Since our LMCa is on the low side of the LMC mass, and our MW is on the high side, our conclusions are conservative in terms of association. The most promising case deserving further evaluation is Dra2 since a more extended LMCa could provide a chance of association at its slightly large angular separation on the sky.

The finding of four confirmed companions of the LMC, with perhaps two more, given the uncertainty in LMC mass, is consistent with the numbers expected for comparable mass systems in LCDM theory Sales et al. (2013).

Recent work from the Gaia Collaboration (Gaia Collaboration et al. 2018b) shows that some of the classical dwarf spheroidals have PMs consistent with being accreted in a group. The analysis of Sales et al. (2011) concluded that none of the classical dwarf spheroidals in the MW would be associated with the Clouds if on a first or second passage. However, with the new PMs, a reanalysis is warranted. An analysis of any new data on the bright classical dwarfs will be presented in a separate work.

We thank the anonymous referee for constructive comments that helped improve the presentation of the results. N.K. is supported by the NSF CAREER award 1455260. L.V. S. acknowledges support from the Hellman Foundation. This work has made use of data from the European Space Agency (ESA) mission Gaia (https://www.cosmos.esa.int/gaia), processed by the Gaia Data Processing and Analysis Consortium (DPAC, https://www.cosmos.esa.int/web/gaia/ dpac/consortium). Funding for the DPAC has been provided by national institutions, in particular, the institutions participating in the Gaia Multilateral Agreement. This project is part of the HSTPROMO (High-resolution Space Telescope PROper MOtion) Collaboration, ${ }^{11}$ a set of projects aimed at improving our dynamical understanding of stars, clusters, and galaxies in the nearby universe through measurement and interpretation of proper motions from HST, Gaia, and other space observatories. We thank the collaboration members for the sharing of their ideas and software.

\section{ORCID iDs}

Nitya Kallivayalil (iD https://orcid.org/0000-0002-3204-1742

Paul Zivick (1) https://orcid.org/0000-0001-9409-3911

Sangmo Tony Sohn (iD https://orcid.org/0000-0001-8368-0221

Roeland P. van der Marel (i) https://orcid.org/0000-0001-

7827-7825

Elena Sacchi (i) https://orcid.org/0000-0001-5618-0109

\section{References}

Ahmed, S. H., Brooks, A. M., \& Christensen, C. R. 2017, MNRAS, 466, 3119 Bechtol, K., Drlica-Wagner, A., Balbinot, E., et al. 2015, ApJ, 807, 50 Besla, G., Kallivayalil, N., Hernquist, L., et al. 2007, ApJ, 668, 949 Bland-Hawthorn, J., \& Gerhard, O. 2016, ARA\&A, 54, 529 Bovy, J., Allende Prieto, C., Beers, T. C., et al. 2012, ApJ, 759, 131 Boylan-Kolchin, M., Besla, G., \& Hernquist, L. 2011, MNRAS, 414, 1560 Bressan, A., Marigo, P., Girardi, L., et al. 2012, MNRAS, 427, 127 Caldwell, N., Walker, M. G., Mateo, M., et al. 2017, ApJ, 839, 20 Conn, B. C., Jerjen, H., Kim, D., \& Schirmer, M. 2018a, ApJ, 857, 70 Conn, B. C., Jerjen, H., Kim, D., \& Schirmer, M. 2018b, ApJ, 852, 68
Deason, A. J., Wetzel, A. R., Garrison-Kimmel, S., \& Belokurov, V. 2015, MNRAS, 453, 3568

D’Onghia, E., \& Lake, G. 2008, ApJL, 686, L61

D’Onghia, E., Springel, V., Hernquist, L., \& Keres, D. 2010, ApJ, 709, 1138

Dooley, G. A., Peter, A. H. G., Carlin, J. L., et al. 2017, MNRAS, 472, 1060 Drlica-Wagner, A., Bechtol, K., Allam, S., et al. 2016, ApJL, 833, L5

Drlica-Wagner, A., Bechtol, K., Rykoff, E. S., et al. 2015, ApJ, 813, 109

Fritz, T. K., Battaglia, G., Pawlowski, M. S., et al. 2018, A\&A, in press (arXiv:1805.00908)

Gaia Collaboration, Brown, A. G. A., Vallenari, A., et al. 2018a, A\&A, 616, A1

Gaia Collaboration, Helmi, A., van Leeuwen, F., et al. 2018b, A\&A, 616, A12

Garrison-Kimmel, S., Boylan-Kolchin, M., Bullock, J. S., \& Lee, K. 2014, MNRAS, 438, 2578

Garrison-Kimmel, S., Wetzel, A., Bullock, J. S., et al. 2017, MNRAS, 471, 1709

Guo, Q., White, S., Boylan-Kolchin, M., et al. 2011, MNRAS, 413, 101

Guo, Q., White, S., Li, C., \& Boylan-Kolchin, M. 2010, MNRAS, 404, 111

Homma, D., Chiba, M., Okamoto, S., et al. 2018, PASJ, 70, S18

Jethwa, P., Erkal, D., \& Belokurov, V. 2016, MNRAS, 461, 2212

Kallivayalil, N., van der Marel, R. P., \& Alcock, C. 2006a, ApJ, 652, 1213

Kallivayalil, N., van der Marel, R. P., Alcock, C., et al. 2006b, ApJ, 638, 772

Kallivayalil, N., van der Marel, R. P., Besla, G., Anderson, J., \& Alcock, C. 2013, ApJ, 764, 161

Kim, D., \& Jerjen, H. 2015, ApJL, 808, L39

Kim, D., Jerjen, H., Geha, M., et al. 2016, ApJ, 833, 16

Kim, D., Jerjen, H., Mackey, D., Da Costa, G. S., \& Milone, A. P. 2015, ApJL, 804, L44

Kirby, E. N., Cohen, J. G., Simon, J. D., et al. 2017, ApJ, 838, 83

Kirby, E. N., Simon, J. D., \& Cohen, J. G. 2015, ApJ, 810, 56

Koposov, S. E., Belokurov, V., Torrealba, G., \& Evans, N. W. 2015a, ApJ, 805,130

Koposov, S. E., Casey, A. R., Belokurov, V., et al. 2015b, ApJ, 811, 62

Koposov, S. E., Walker, M. G., Belokurov, V., et al. 2018, MNRAS, 479, 5343

Laevens, B. P. M., Martin, N. F., Bernard, E. J., et al. 2015a, ApJ, 813, 44

Laevens, B. P. M., Martin, N. F., Ibata, R. A., et al. 2015b, ApJL, 802, L18

Li, T. S., Simon, J. D., Drlica-Wagner, A., et al. 2017, ApJ, 838, 8

Li, T. S., Simon, J. D., Pace, A. B., et al. 2018, ApJ, 857, 145

Li, Y.-S., \& Helmi, A. 2008, MNRAS, 385, 1365

Lynden-Bell, D. 1976, MNRAS, 174, 695

Martin, N. F., Geha, M., Ibata, R. A., et al. 2016, MNRAS, 458, L59

Martin, N. F., Nidever, D. L., Besla, G., Olsen, K., et al. 2015, ApJL, 804, L5

Massari, D., \& Helmi, A. 2018, A\&A, in press (arXiv:1805.01839)

McConnachie, A. W. 2012, AJ, 144, 4

Moster, B. P., Naab, T., \& White, S. D. M. 2013, MNRAS, 428, 3121

Nidever, D. L., Majewski, S. R., Butler Burton, W., \& Nigra, L. 2010, ApJ, 723, 1618

Patel, E., Besla, G., \& Sohn, S. T. 2017, MNRAS, 464, 3825

Sales, L. V., Navarro, J. F., Abadi, M. G., \& Steinmetz, M. 2007, MNRAS, 379,1464

Sales, L. V., Navarro, J. F., Cooper, A. P., et al. 2011, MNRAS, 418, 648

Sales, L. V., Navarro, J. F., Kallivayalil, N., \& Frenk, C. S. 2017, MNRAS, 465, 1879

Sales, L. V., Wang, W., White, S. D. M., \& Navarro, J. F. 2013, MNRAS, 428,573

Simon, J. D. 2018, ApJ, 863, 89

Simon, J. D., Drlica-Wagner, A., Li, T. S., et al. 2015, ApJ, 808, 95

Simon, J. D., Li, T. S., Drlica-Wagner, A., et al. 2017, ApJ, 838, 11

Sohn, S. T., Anderson, J., \& van der Marel, R. P. 2012, ApJ, 753, 7

Sohn, S. T., Besla, G., van der Marel, R. P., et al. 2013, ApJ, 768, 139

Springel, V., Wang, J., Vogelsberger, M., et al. 2008, MNRAS, 391, 1685

Torrealba, G., Belokurov, V., Koposov, S. E., et al. 2018, MNRAS, 475, 5085

Torrealba, G., Koposov, S. E., Belokurov, V., et al. 2016a, MNRAS, 463, 712

Torrealba, G., Koposov, S. E., Belokurov, V., \& Irwin, M. 2016b, MNRAS, 459, 2370

van der Marel, R. P., Alves, D. R., Hardy, E., \& Suntzeff, N. B. 2002, AJ, 124, 2639

Vogelsberger, M., Genel, S., Springel, V., et al. 2014, Natur, 509, 177

Walker, M. G., Mateo, M., Olszewski, E. W., et al. 2016, ApJ, 819, 53

Watkins, L. L., van der Marel, R. P., Sohn, S. T., \& Evans, N. W. 2018 , arXiv: 1804.11348

Wetzel, A. R., Deason, A. J., \& Garrison-Kimmel, S. 2015, ApJ, 807, 49

Yozin, C., \& Bekki, K. 2015, MNRAS, 453, 2302

\footnotetext{
11 http://www.stsci.edu/ marel/hstpromo.html
} 